\title{
Coordinated Dynamics Control of a Free-Floating Dual-arm Space Robot
}

\author{
Hongcai Zhao (Corresponding author) \\ School of Automatic Engineering, Qingdao Technological University \\ 11 Fushun road, Qingdao 266033, China \\ E-mail: zhaohongcai2003@163.com \\ Congqing Wang \\ College of Automation, Nanjing University of Aeronautics and Astronautics \\ 8 Lifu Street, Nanjing 210016, China \\ E-mail: wangcq@nuaa.edu.cn \\ Zhen Guo \\ School of Automatic Engineering, Qingdao Technological University \\ 11 Fushun road, Qingdao 266033, China \\ E-mail: qs2004b@163.com
}

\begin{abstract}
The dynamics control of a dual-arm space robot installed on a free-flying spacecraft without base position and orientation control holding a single object is discussed in this paper. The movement of two arms and the object seriously affects position and orientation of the base and the robot grasp programming later due to heavy coupling between the arms and the base. The closed kinematic chain constrains and the Generalized Jacobian Matrix (GJM) of the dual-arm space robot are built. In order to save energy consumption, a coordinated dynamics control scheme with zero internal forces control based on the well-known computed torque method is used to control the trajectory of a grasped object, in which the disturbance to the base can be effectively decreased. The validity of this method is proved by computer simulations for a grasp system with a planar dual-arm space robot of three freedoms.
\end{abstract}

Keywords: Dual-arm space robot, Generalized Jacobian Matrix (GJM), Coordinated control

\section{Introduction}

In recent years, coordinated control of multiple-arm space robot systems has received considerable attention, because the utilization of the coordinated multiple-arm system provides greater lifting and manipulation capability as well as higher flexibility in space tasks. The cooperation manipulation increases the possibility for space robots to carry out more complicated and dexterous tasks, which may not be accomplished by a single space robot. There are several approaches to the multiple-arm space robot. Kazuya Yoshida et al. (1991, p.2516-2521) presented the control problem of multiple manipulators installed on a free-flying space robot, in which a method to control two arms simultaneously: one arm traces a given path, while the other arm works both to keep the satellite attitude .Yan-Ru Hu and George Vukovich (1998, p. 217-230) presented the position / force control of coordinated robots mounted on spacecraft manipulating an object with closed kinematic chain constraints. The Generalized Jacobian Matrix (GJM) is formulated for the motion control of the multi-arm system. S.A.Moosavian and E. Papadopoulos (1997, p.853-858) and S. Ali A et al (1997, p.10-12.) suggested a multiple impedance control (MIC) algorithm for space robotic systems in which manipulators are mounted on a free-flying base. It was shown that under the MIC algorithm, all participating manipulators, the free-flyer base, and the manipulated object exhibited the same designated impedance behavior. S.H.Murphy et al. (1991, p. 468-478) presented the dynamic equations of motion for two or more cooperating manipulators on a freely moving mobile platform. The equations of motion were shown to be identical in structure to the fixed-platform cooperative manipulator dynamics. A.K.Swain and A.S.Morris (2004, p.271-283) described the control of multi-arm cooperating manipulator systems handing a common object. Inverse dynamics controllers with motive force compensation were developed for the cooperating fixed-base, free-floating, and free-flying space 
manipulator system. In this paper, kinematics of a dual-arm space robot and the relationship with closed constrains between the end-effects of the space robot and the object are obtained. In space operations it is expected that internal force will be minimal to save energy consumption, hence a coordinated control scheme with zero internal force control is presented. Simulation results for a planar dual-arm space robot show that errors of the position and force asymptotically converge to zero.

\section{The closed chained constrains and generalized Jacobian matrix}

A grasp system consisting of a free-floating space dual -arm robot, spacecraft and the object is shown in Fig.1.

In order to describe the motion of the grasp system, coordinate systems are defined as:

$\sum_{0}$ : The coordinate system fixed at the mass center of the spacecraft ;

$\sum_{I}$ : The inertial coordinate system fixed at the mass center of the coordinate system;

$\sum_{j i}:$ The link coordinate system fixed at the i-th joint of the arm;

$m_{0}, I_{0}:$ mass and inertial of spacecraft;

$m_{j}^{i}, \boldsymbol{I}_{j}^{i}$ :mass and inertial of the $\mathrm{i}$-th link of the $\mathrm{j}$-th arm;

$m_{c}, I_{c}:$ mass and inertial of the grasped object;

$\boldsymbol{r}_{0}, \boldsymbol{r}_{j c}^{i}, \boldsymbol{r}_{c}$ position vectors of all links from the

Origin of the coordinate system to the origin of the link system, mass center of all links and the load;

${ }^{i} \boldsymbol{p}_{j}$ : position vector of $\mathrm{i}$-th link from the origin of $\mathrm{i}$-th link to the mass center at the $\mathrm{j}$-th arm;

${ }_{i}^{i-1} I_{j}$ :position vector from the origin of the link system of the (i-1) th link to the origin of the link system of the i-th link expressed in the link system of the (i-1) th link of the $\mathrm{j}$-th arm;

$\dot{\boldsymbol{q}}_{j}^{i}:$ joint velocity vector of the $\mathrm{j}$-th arm;

${ }^{i} \boldsymbol{\omega}_{j}$ : angular velocities of the $\mathrm{i}$-th link of the $\mathrm{j}$-th arm expressed in $\sum_{j i}$ system;

${ }^{i} \boldsymbol{V}_{j}$ : linear velocity of the i-th link of the j-th expressed in $\sum_{j i}$ system;

${ }^{i} \boldsymbol{\omega}_{j c}$ : angular velocity of the mass center of the ith link of the $\mathrm{j}$-th arm expressed in $\sum_{j i}$ system,

where $j=L, R$ are defined as left arm and right arm which have same the numbers of the freedom; $i=1 \cdots n, n$ is the numbers of freedom for the $\mathrm{j}$-th arm.

The recursive relationships for all links are as follows

$$
\left\{\begin{array}{l}
{ }^{i} \boldsymbol{\omega}_{j}={ }_{i-1}^{i} \boldsymbol{R}_{j}{ }^{i-1} \boldsymbol{\omega}_{j}+\dot{\boldsymbol{q}}_{j}^{i}{ }^{i} z_{i} \\
{ }^{i} \boldsymbol{V}_{j}={ }_{i-1}^{i} \boldsymbol{R}_{j}\left({ }^{i-1} \boldsymbol{V}_{j}+{ }^{i-1} \boldsymbol{\omega}_{j} \times{ }_{i}^{i-1} \boldsymbol{J}_{j}\right)
\end{array}\right.
$$

Where ${ }^{i} z_{i}$ is an identity vector of $\mathrm{z}$ axis in $\sum_{j i}$ coordinate. An operator ' $X$ ' for

$$
r=\left[r_{x}, r_{y}, r_{z}\right]^{T}
$$

The velocity of the $\mathrm{i}$-th link of the $\mathrm{j}$-th arm can be expressed recursively based on Kazuya Yoshida et al. (991,p.2516-2521) as follows:

$$
{ }^{i} \boldsymbol{V}_{j}=\boldsymbol{\Phi}_{j}(i, i-1)^{i-1} \boldsymbol{V}_{j}+{ }^{i} n_{j} \dot{\boldsymbol{q}}_{j}^{i} \quad j=R, L
$$

Where ${ }^{i} \boldsymbol{V}_{j}=\left[{ }^{i} \boldsymbol{V}_{j}{ }^{T},{ }^{i} \boldsymbol{\omega}_{j}{ }^{T}\right]^{T}, \boldsymbol{\varphi}_{j}(i, i-1)=\left[\begin{array}{cc}{ }_{i-1}^{i} \boldsymbol{R}-{ }_{i-1}^{i} \boldsymbol{R}^{i-1} \boldsymbol{l}_{j} \times \\ \boldsymbol{O} & { }_{i=1}^{i} \boldsymbol{R}_{j}\end{array}\right] \in R^{6 \times 6},{ }^{i} \boldsymbol{n}_{j}=[0,0,0,0,0,1]^{T}$

The velocity of end-effectors for $\mathrm{j}$-th arm expressed in the $\mathrm{j}$-th link frame is represented as 


$$
\begin{aligned}
{ }^{n+1} V_{j} & =\phi_{j}(n+1, n)^{n} V_{j} \\
& =\phi_{j}(n+1,0) V_{0}+\phi_{j}(n+1, n) G_{j} N_{j} \dot{q}_{j}
\end{aligned}
$$

Where $V_{0}=\left[v_{0}{ }^{T}, \omega_{0}{ }^{T}\right]^{T}, V_{c}=\left[v_{c}{ }^{T}, \omega_{c}{ }^{T}\right]^{T}$,

$\dot{\boldsymbol{q}}_{j}=\left[\dot{\boldsymbol{q}}_{j}^{1} \cdots \dot{\boldsymbol{q}}_{j}^{n}\right], \boldsymbol{G}_{j}=\left[\boldsymbol{\varphi}_{j}(n, 1) \cdots \boldsymbol{\varphi}_{j}(n, n)\right], \boldsymbol{N}_{j}=\operatorname{di} \operatorname{ag}\left(\boldsymbol{n}_{j} \cdots^{n} \boldsymbol{n}_{j}\right)$.

The velocity of $\mathrm{j}$-th arm expressed in the j-th link frame is described as

$$
\dot{X}_{e j}=J_{b j} V_{0}+J_{j} \dot{q}_{j}
$$

Where $\boldsymbol{J}_{b j}=\boldsymbol{R}_{e j} \boldsymbol{\Phi}_{j}(n+1,0), \boldsymbol{J}_{j}=\boldsymbol{R}_{e j} \boldsymbol{\Phi}_{j}(n+1, n) \boldsymbol{G}_{j} \boldsymbol{N}_{j}, R_{e j}=\operatorname{di} \operatorname{ag}\left({ }_{n+1}^{0} R_{j},{ }_{n+1}^{0} R_{j}\right) .{ }_{n+1}^{0} R_{j}$ is a rotation matrix from the end-effectors frame to the spacecraft frame. The linear and angular velocities of the mass center of the ith link of the $\mathrm{j}$-th arm are expressed as

$$
\left[\begin{array}{c}
{ }^{i} v_{j c} \\
{ }^{i} \omega_{j c}
\end{array}\right]=\left[\begin{array}{cc}
E & -{ }^{i} p_{j}{ }^{\circ} \\
0 & E
\end{array}\right]\left[\begin{array}{c}
{ }^{i} v_{j} \\
{ }^{i} \omega_{j}
\end{array}\right]
$$

Where ${ }^{i} v_{j c},{ }^{i} \omega_{j c},{ }^{i} v_{j}$ and ${ }^{i} \omega_{j}$ are the linear and angular velocities of the mass center of the i-th link expressed in the i-th link frame for the j-th arm. $E \in R^{3 \times 3}$ is an identity matrix, and ${ }^{i} P_{j}$ is the vector from the origin of the ith link frame to the mass center of the ith link and expressed in the ith link frame for the $\mathrm{j}$-th arm.

From Eq. (5), the linear and angular velocities of the mass center of the ith link of the $j$-th arm expressed in the spacecraft frame can be described as

$V_{j c}^{i}=S_{j}^{i}{ }_{j}^{i} V_{j}$

Where $V_{j c}^{i}=\left[v_{j c}^{i}{ }^{i} \omega_{j c}^{i}{ }^{T}\right]^{T},{ }^{i} V_{j}=\left[{ }^{i} v_{j}^{T i} \omega_{j}^{T}\right]^{T}, S_{j}^{i}=\left[\begin{array}{cc}{ }_{i}^{0} R_{j} & -{ }_{i}^{0} R_{j}{ }^{i} p_{j} \times \\ 0 & { }_{i}^{0} R_{j}\end{array}\right],{ }_{i}^{0} R_{j}$ is a rotation matrix from the ith link frame to the spacecraft frame.

The velocities of all links and their mass centers of the $\mathrm{j}$-th arm can be described as

$V_{j}=\Phi_{j} N_{j} \dot{q}_{j}+D_{j} V_{0}$

$V_{j c}=S_{j} V_{j}$

Where $\quad V_{j}=\left[V_{j}^{V^{T}} \cdots V_{j}^{n^{T}}\right]^{T}, V_{j c}=\left[V_{j c}^{V^{T}} \cdots V_{j c}^{n^{T}}\right]^{T}, \dot{q}_{j}=\left[\dot{q}_{j}^{1} \cdot \cdots \dot{q}_{j}^{n}\right]^{T} \quad, S_{j}=\operatorname{diag}\left(S_{j}^{1} \cdots S_{j}^{n}\right) \quad, \quad \boldsymbol{D}_{j}=\left[\boldsymbol{\varphi}_{j}^{T}(1,0) \cdots \boldsymbol{\varphi}_{j}^{T}(n, 0)\right]^{T} \quad$,

$$
\Phi_{j}=\left[\begin{array}{cccc}
\boldsymbol{\varphi}_{j}(1,1) & 0 & \cdots & 0 \\
\boldsymbol{\varphi}_{j}(2,1) & \boldsymbol{\varphi}_{j}(2,2) & \cdots & 0 \\
\vdots & \vdots & \ddots & \vdots \\
\boldsymbol{\varphi}_{j}(n, 1) & \boldsymbol{\varphi}_{j}(n, 2) & \cdots & \boldsymbol{\varphi}_{j}(n, n)
\end{array}\right]
$$

We assume that the coordinated space robotic system is operated in free-floating mode without external forces and moments exerted on the grasp system, so the total linear and angular momenta are conserved respectively. Furthermore, we assume the total linear and angular momenta are zero. The relationship between the joint and spacecraft velocities is obtained as

$V_{0}=J_{s} \dot{q}$

Where $\quad \boldsymbol{J}_{s}=-\left(\hat{\boldsymbol{M}}_{0}+\boldsymbol{M}_{m} \boldsymbol{S} \boldsymbol{D}+\hat{\boldsymbol{M}}_{c} \boldsymbol{J}_{c b}\right)^{-1}\left(\boldsymbol{M}_{m} \boldsymbol{S} \Phi \boldsymbol{N}+\hat{\boldsymbol{M}}_{c} \boldsymbol{J}_{c q}\right) \quad, \quad \hat{\boldsymbol{M}}_{0}=\left[\begin{array}{cc}m_{0} \boldsymbol{E}_{3} & 0 \\ m_{0} \boldsymbol{r}_{0}^{\times} & \boldsymbol{J}_{0}\end{array}\right] \in \boldsymbol{R}^{6 \times 6}$ $M_{m}=\left[M_{m L} \cdots M_{m R}\right], \quad \boldsymbol{M}_{i j}=\left[\left[\begin{array}{cc}m_{j}^{1} \boldsymbol{E}_{3} & 0 \\ m_{j}^{1} \boldsymbol{r}_{j c}^{1^{\times}} & \boldsymbol{I}_{j}^{1}\end{array}\right] .\left[\begin{array}{cc}m_{j}^{n} \boldsymbol{E}_{3} & 0 \\ m_{j}^{n} \boldsymbol{r}_{j c}^{n^{\times}} & \boldsymbol{I}_{j}^{n}\end{array}\right]\right] \in R^{6 \times 6 n ;} \quad \hat{\boldsymbol{M}}_{c}=\left[\begin{array}{ccc}m_{c} \boldsymbol{E}_{3} & \boldsymbol{O} \\ m_{c} \boldsymbol{r}_{c}^{\times} & \boldsymbol{I}_{c}\end{array}\right] \in R^{6 \times 6}, \quad \boldsymbol{D}=\left[\boldsymbol{D}_{L}^{T}, \boldsymbol{D}_{R}^{T}\right]^{T}$, $\boldsymbol{N}=\operatorname{di} \operatorname{ag}\left(\boldsymbol{N}_{L}, \boldsymbol{N}_{R}\right) \quad . \quad \boldsymbol{J}_{c b}=\frac{1}{2} \boldsymbol{J}_{c} \boldsymbol{J}_{b}, \boldsymbol{J}_{c q}=\frac{1}{2} \boldsymbol{J}_{c} \boldsymbol{J}_{q}, \boldsymbol{J}_{c}=\left[\boldsymbol{J}_{c L L}^{-1}, \boldsymbol{J}_{c e R}^{-1}\right], \quad \boldsymbol{J}_{b}=\left[\boldsymbol{J}_{b L}^{T}, \boldsymbol{J}_{b R}^{T}\right]^{T}$, $\boldsymbol{J}_{q}=\operatorname{diag}\left(\boldsymbol{J}_{L}, \boldsymbol{J}_{R}\right) . \boldsymbol{\Phi}$ is a transformation matrix, and $\boldsymbol{S}, \boldsymbol{D}$, and $\boldsymbol{N}$ are relevant matrices, $\dot{\boldsymbol{q}}$ is the velocity vector of all robot joints.

In terms of the Eq. (4), the relationship between the object, spacecraft, and joint velocities is described as 
$\dot{X}_{e}=J_{b} V_{0}+J_{q} \dot{q}$

Substituting Eq. (9) into Eq. (10). The closed chain constrains relationship between end-effectors and joint velocities is expressed as follows

$\dot{X}_{e}=J_{b} J_{s} \dot{q}+J_{q} \dot{q}=J_{e} \dot{q}$

Where $J_{e}=J_{b} J_{s}+J_{q}$ is defined as the generalized Jacobian matrix (GJM) of the free-floating space dual-arm robot, in which $\mathcal{J}_{s}$ is related to dynamics parameters of all arms, spacecraft and payload, $\boldsymbol{J}_{q}=\operatorname{diag}\left(\boldsymbol{J}_{L}, \boldsymbol{J}_{R}\right)$ is a conventional Jacobian matrix of fixed-base.

\section{Dynamics coordinated control}

\subsection{Dynamics equation}

Dynamics equation for free-floating space dual-arms is described based on Yan-Ru Hu et al. (1998, p. 217-230), S.A.Moosavian et al (1997, p.853-858) and S. Ali A et al (1997, p.10-12.) as follows

$$
M \ddot{q}+\boldsymbol{C}=\boldsymbol{T}-\boldsymbol{J}^{T} \boldsymbol{F}
$$

where $\boldsymbol{M}=\boldsymbol{N}^{T} \boldsymbol{\phi}^{T}\left(\boldsymbol{M}_{q}^{-1}+\boldsymbol{D} \boldsymbol{M}_{0}^{-1} \boldsymbol{D}^{T}\right)^{-1} \boldsymbol{\phi} \boldsymbol{N}$

$$
\begin{aligned}
& \boldsymbol{C}=\boldsymbol{N}^{T} \boldsymbol{\Phi}^{T}\left(\boldsymbol{M}_{q}^{-1}+\boldsymbol{D} \boldsymbol{M}_{0}^{-1} \boldsymbol{D}^{T}\right)^{-1}\left[\boldsymbol{M}_{q}^{-1} \boldsymbol{b}--\boldsymbol{D} \boldsymbol{M}_{0}^{-1} \boldsymbol{b}_{0}+\left(\boldsymbol{\phi} \boldsymbol{N}+\dot{\boldsymbol{D}}{ }_{s}\right) \dot{\boldsymbol{q}}\right] \\
& \boldsymbol{J}^{T}=\boldsymbol{N}^{T} \boldsymbol{\Phi}^{T}\left(\boldsymbol{M}_{q}^{-1}+\boldsymbol{D} \boldsymbol{M}_{0}^{-1} \boldsymbol{D}^{T}\right)^{-1} \boldsymbol{M}_{q}^{-1} \boldsymbol{B}
\end{aligned}
$$

with $\boldsymbol{M}_{o}=\left[\begin{array}{cc}m_{o} E_{3} & 0 \\ \boldsymbol{O} & \boldsymbol{I}_{o}\end{array}\right] \in R^{6 \times 6}, \boldsymbol{b}_{0}=\left[\boldsymbol{O}\left(\boldsymbol{\omega}_{0} \times \boldsymbol{I}_{0} \boldsymbol{\omega}_{0}\right)^{T}\right]^{T}, \boldsymbol{\omega}_{0}$ is an angular velociy expressed in spacecraft frame , $\boldsymbol{T}$ is the vector of joint driving torque , $\boldsymbol{M}_{q}=\operatorname{diag}\left[\boldsymbol{M}_{L}, \boldsymbol{M}_{R}\right]$, $\left.{ }^{i} \boldsymbol{M}=\left[\begin{array}{cc}m_{j}^{j} E_{3} & -m_{j}^{i} \boldsymbol{p}_{j}^{\times} \\ m_{j}^{i} \boldsymbol{p}_{j}^{\times} & \boldsymbol{I}_{j}^{i}\end{array}\right] \in R^{6 \times 6}, \boldsymbol{M}_{j}=\operatorname{di} \operatorname{ag}\left[\boldsymbol{M}_{j}{ }^{1}{ }^{n} \boldsymbol{M}\right], \boldsymbol{b}_{j}={ }^{-1} \boldsymbol{b}_{j} \cdot{ }^{n} \boldsymbol{b}_{j}\right]^{T},{ }^{i} \boldsymbol{b}_{j}=\left[\begin{array}{c}m_{j}^{i}\left({ }^{i} \boldsymbol{\omega}_{j}^{\times}\right)\left({ }^{i} \boldsymbol{\omega}_{j}^{\times}\right)^{i} \boldsymbol{p}_{j} \\ \left({ }^{i} \boldsymbol{\omega}_{j}^{\times}\right) I_{j}^{i}\left({ }^{i} \boldsymbol{\omega}_{j}^{\times}\right)\end{array}\right], \boldsymbol{b}=\left[\boldsymbol{b}_{L}^{T}, \boldsymbol{b}_{R}^{T}\right]^{T}$, $\boldsymbol{q}$ is the vector of joint coordinate, $F=\left[F_{L}{ }^{T}, F_{R}{ }^{T}\right]^{T}$ is the force vector exerted on the object by end-effectors of the dual- arms, and $\left.\boldsymbol{F}_{j}=\left[\boldsymbol{f}_{j}^{T} \boldsymbol{\eta}_{j}^{T}\right]^{T}, \boldsymbol{f}_{j}=\left[\boldsymbol{f}_{j x}, \boldsymbol{f}_{j y}, \boldsymbol{f}_{j z}\right]^{T}, \boldsymbol{\eta}_{j}=\boldsymbol{\eta}_{j x} \boldsymbol{\eta}_{j y} \boldsymbol{\eta}_{j z}\right]^{T} \quad(j=L, R)$.

$\boldsymbol{J}$ is the force Jacobian matrix for the end force, ${ }^{i} \boldsymbol{\omega}_{j}$ is the angular velocity of $\mathrm{i}$-th link frame to the spacecraft frame, and ${ }^{i} \boldsymbol{\omega}_{j}{ }^{\times}$is ${ }^{i} \boldsymbol{\omega}_{j}$ inverse symmetry matrix, $\boldsymbol{B}=\left[\boldsymbol{O} \cdots \boldsymbol{O} \boldsymbol{\varphi}_{j}(n+1, n)\right]^{T}$.

According to Newton-Euler equation, dynamics equation of the object can be described as follows $\boldsymbol{M}_{c}\left(\boldsymbol{X}_{c}\right) \ddot{\boldsymbol{X}}_{c}+\boldsymbol{C}_{c}\left(\boldsymbol{X}_{c}, \dot{\boldsymbol{X}}_{c}\right)=\boldsymbol{J}_{c e}{ }^{T} \boldsymbol{F}=F_{O}$

Where $\boldsymbol{M}_{c}=\left[\begin{array}{cc}m_{c} \boldsymbol{E}_{3} & \boldsymbol{O} \\ \boldsymbol{O} & \boldsymbol{I}_{c}\end{array}\right] \in R^{6 \times 6}, \ddot{X}_{c}=\left[\ddot{r}_{c}^{T}, \dot{\omega}^{T}\right]^{T} \in R^{6}, \boldsymbol{C}_{c}=\left[\boldsymbol{O}\left(\boldsymbol{\omega}_{c} \times \boldsymbol{I} \boldsymbol{\omega}_{c}\right)^{T}\right]^{T} . \boldsymbol{\omega}_{c}$ is velocity for the object, $\boldsymbol{F}$ is the force of end-effect exerted on the object. $F_{o}=\left[f_{o}^{T}, N_{o}^{T}\right]^{T} \in R^{6}, E_{3} \in R^{3 \times 3}$ is an identity matrix. $f_{o}, N_{o}$ are the resultant force and moment applied to the object by dual arms.

3.2 Dynamics coordinated control

The end effectors force can be obtained by using a pseudo inverse technique. The result of computation is written as

$\boldsymbol{F}=\left(\boldsymbol{J}_{c e}{ }^{T}\right)^{+} F_{0}+\left(E_{6}-\left(\boldsymbol{J}_{c e}{ }^{T}\right)^{+}\left(\boldsymbol{J}_{c e}{ }^{T}\right)\right) \lambda=F_{m}+F_{I}$

Where $\left(\boldsymbol{J}_{c e}{ }^{T}\right)^{+}=\boldsymbol{J}_{c e}\left(\boldsymbol{J}_{c e}{ }^{T} \boldsymbol{J}_{c e}\right)^{-1}, F_{I}=\left(E_{6}-\left(\boldsymbol{J}_{c e}{ }^{T}\right)^{+}\left(\boldsymbol{J}_{c e}{ }^{T}\right)\right) \lambda$ is defined as an internal force which does not contribute any driving force to the object, $F_{I}=\left[{F_{I L}}^{T}, \boldsymbol{F}_{I R}{ }^{T}\right]^{T} \in R^{12 \times 1}$, and $\lambda \in R^{6}$ is any vector in the null-space of $J_{c e}{ }^{T}$, that is $\boldsymbol{J}_{c e}{ }^{T} \boldsymbol{F}_{I}=\boldsymbol{O}, F_{m}$ is a manipulating force, $F_{m}=\left(\boldsymbol{J}_{c e}{ }^{T}\right)^{+} F_{o}, E_{6} \in R^{6 \times 6}$ is an identity matrix, and $\left.\boldsymbol{F}_{I j}=\left[\boldsymbol{f}_{I j}^{T} \boldsymbol{\eta}_{l j}^{T}\right]^{T}, \boldsymbol{f}_{I j}=\left[\boldsymbol{f}_{I j x}, \boldsymbol{f}_{I j y}, \boldsymbol{f}_{I j z}\right]^{T}, \boldsymbol{\eta}_{I j}=\boldsymbol{\eta}_{I j x}, \boldsymbol{\eta}_{I j y} \boldsymbol{\eta}_{I j z}\right]^{T}$. However in space operations it is expected that a targeted object will be grabbed with a special tool or gripper. In such cases, it is expected that internal forces and moments will be minimal and hence, $F_{I}$ can be chosen equal to zero. 
Assume that all the end-effectors are firmly holding the object at the contact point so that there is no relative motion between the object and any end-effectors. We have the relationship:

$\boldsymbol{J}_{e} \dot{\boldsymbol{q}}=\boldsymbol{J}_{c e} \dot{\boldsymbol{X}}$

Assume that all robot manipulators work in nonsingular work space and $J_{e}$ is a nonsingular matrix, we also have

$\ddot{\boldsymbol{q}}=\boldsymbol{J}_{e}^{-1}\left(\dot{\boldsymbol{J}}_{c e} \dot{\boldsymbol{X}}_{c}+\boldsymbol{J}_{c e} \ddot{\boldsymbol{X}}_{c}-\dot{\boldsymbol{J}}_{e} \dot{\boldsymbol{q}}\right)$

With the above relations, substituting Eq. (14) and Eq. (16) into Eq. (12), the combined dynamics equation of the entire grasp system for the free-floating space robot is obtained as

$\boldsymbol{A}_{c} \ddot{\boldsymbol{X}}_{c}+\boldsymbol{B}_{c}=\boldsymbol{T}-\boldsymbol{J}^{T} \boldsymbol{F}_{I}$

Where $\boldsymbol{A}_{c}=\boldsymbol{M}_{e}^{-1} \boldsymbol{J}_{c e}+\boldsymbol{J}^{T}\left(\boldsymbol{J}_{c e}{ }^{T}\right)^{+} \boldsymbol{M}, \quad \boldsymbol{B}_{c}=\boldsymbol{M}_{e}^{-1}\left(\boldsymbol{j}_{c e} \dot{\boldsymbol{X}}_{c}-\boldsymbol{j}_{e} \dot{q}\right)+\boldsymbol{C}+\boldsymbol{J}^{T}\left(\boldsymbol{J}_{c e}{ }^{T}\right)^{+} \boldsymbol{C}_{c}$.

Let $\boldsymbol{A}_{c}, \boldsymbol{B}_{c}$ can be exactly estimated, the following coordinated control law is proposed

$\tau=\tau_{p}+\tau_{i}$

Where $\tau_{p}, \tau_{i}$ are position control for the object and internal force control for the dual-arms respectively, and they can be chosen as

$T_{p}=\boldsymbol{A}_{c}\left(\ddot{X}_{c}^{d}+\boldsymbol{K}_{v}^{p}\left(\dot{X}_{c}^{d}-\dot{X}_{c}\right)+K_{p}^{p}\left(\boldsymbol{X}_{c}^{d}-\boldsymbol{X}_{c}\right)\right)+\boldsymbol{B}_{c}$

$\tau_{i}=\boldsymbol{J}^{T}\left(\boldsymbol{F}_{I}^{d}+\boldsymbol{K}_{i}^{i} \int\left(\boldsymbol{F}_{I}^{d}-\boldsymbol{F}_{I}\right) d t\right)$

Where $\boldsymbol{X}_{c}^{d}, \dot{\boldsymbol{X}}_{c}^{d}, \ddot{\boldsymbol{X}}_{c}^{d}$ are the desired position, velocity and acceleration vectors of the object

Respectively; $\boldsymbol{X}_{c}, \dot{\boldsymbol{X}}_{c}$ are actual position and velocity vectors for the object; $\boldsymbol{F}_{I}^{d}, \boldsymbol{F}_{I}$ are the desired force and actual internal force vectors respectively. $\boldsymbol{K}_{v}^{p}, \boldsymbol{K}_{p}^{p}$ are feedback control gain matrices, $K_{i}^{i}$ is an integral gain matrix. $J_{e} \in R^{12 \times 12}$ is a nonsingular matrix.

Substituting the coordinated control law given by Eq. (18) into the combined dynamics equation of the entire grasp system for the free-floating space robot, we can obtain

$\boldsymbol{A}_{c}\left(\ddot{e}_{p}+\boldsymbol{K}_{v}^{p} \dot{\boldsymbol{e}}_{p}+\boldsymbol{K}_{p}^{p} \boldsymbol{e}_{p}\right)=\boldsymbol{J}^{T}\left(\boldsymbol{e}_{f}+\boldsymbol{K}_{i}^{i} \int \boldsymbol{e}_{f} d t\right)$

Where $\boldsymbol{e}_{p}=x_{c}^{d}-x_{c}, \quad \dot{\boldsymbol{e}}=\ddot{x}_{c}^{d}-\dot{x}_{c}, \ddot{\boldsymbol{e}}_{p}=\ddot{x}_{c}^{d}-\ddot{x}_{c}, \boldsymbol{e}_{I}=F_{I}^{d}-F_{I}$.

Pr-multiplying (21) by $\boldsymbol{J}_{c e}{ }^{T} J^{T}$, and using the facts that $\boldsymbol{J}_{c e}{ }_{c e}^{T} \boldsymbol{e}_{I}=\boldsymbol{J}_{c e}{ }^{T}\left(F_{I}^{d}-F_{I}\right)=0$, we can obtain

$\ddot{\boldsymbol{e}}_{p}+\boldsymbol{K}_{v}^{p} \dot{\boldsymbol{e}}_{p}+\boldsymbol{K}_{p}^{p} \boldsymbol{e}_{p}=\mathbf{O}$

Because of the choice of $\boldsymbol{K}_{v}^{p}, \boldsymbol{K}_{p}^{p}$, it can be concluded that $\boldsymbol{e}_{p}$ and $\dot{\boldsymbol{e}}_{p}$ converge to zero as $t \rightarrow \infty$.

Substituting (22) into (21), and using $J$ and $J_{e}$ are non-singular, error equation of internal forces can be written as

$\boldsymbol{e}_{f}+\boldsymbol{K}_{i}^{i} \int \boldsymbol{e}_{f} d t=0$

For any positive definite and symmetric matrix $\boldsymbol{K}_{i}^{i}$, error for internal force converges to zero as $t \rightarrow \infty$. Hence the coordinated control law guarantees the asymptotic stability and convergence for both position and internal force control.

\section{Simulation}

A simulation for a planar dual-arm space robot mounted on a spacecraft performing a cooperative manipulation task is done to show effectiveness of the proposed coordinated control scheme in case of where the dynamics parameters of the object and robot arms are exactly known. In the simulation, the planar dual-arms carry one object as shown in Fig 5. The parameters for the grasp system are as follows:

$m_{0}=100 \mathrm{~kg}, m_{1}=15 \mathrm{~kg}, m_{2}=10 \mathrm{~kg}, m_{3}=5 \mathrm{~kg}, m_{c}=10 \mathrm{~kg}, l_{1}=l_{2}=l_{3}=1 \mathrm{~m} I_{0}=200 \mathrm{kgm}^{2}$, 
$I_{1}=0.3 \mathrm{kgm}^{2}, I_{2}=0.2 \mathrm{kgm}^{2}, I_{3}=0.1 \mathrm{kgm}^{2}, I_{c}=15 \mathrm{kgm}^{2}$. The distances from the object mass center to two end-effectors are $0.5 \mathrm{~m}$ respectively. The distances from the mass center of the spacecraft to the first joints of two arms are $1.5 \mathrm{~m}$ respectively. The desired trajectories for the object are given as

$\left\{\begin{array}{l}X_{c x}^{d}(t)=1.5+0.2 \cos (2 t)(\mathrm{m}) \\ X_{c y}^{d}(t)=1.0+0.2 \cos (2 t)(\mathrm{m}) \\ X_{c \theta}^{d}=0.05 \cos (2 t)(\mathrm{rad})\end{array}\right.$

Where the initial position of the object is $(1.5,1.0,0)(\mathrm{m})$, the initial position of the spacecraft is $(1.5,0,0)(\mathrm{m})$. Control gain matrices are chosen as $K_{v}^{p}=6.7 E_{3}, K_{p}^{p}=100 E_{3}, K_{i}^{i}=1.2 E_{3}$. The initial internal force is chosen as $F_{I}=[5,0,0,-5,0,0]^{T}(N)$, the desired zero internal force is chosen as $F_{I}^{d}=[0,0,0,0,0,0]^{T}(N)$. The sampling period is 10 ms. The tracking trajectories of the object are shown in Fig.2, and the movement of the spacecraft is shown in Fig.3. The internal force responses are shown in Fig.4. Motion responses of dual-arms, object and spacecraft are shown in Fig.5. From Fig.3, it is seen that the coordinated control scheme can effectively reduce the disturbance to the spacecraft.

\section{Conclusion}

A coordinated control scheme for a free floating space dual-arm robot has been developed. The position/ force hybrid control with zero internal force was proposed for the first time. Unlike previous coordinated control schemes, zero internal force control for the space robots can save energy consumption throughout the operation. The computation simulation results have shown the control objective was achieved successfully.

\section{References}

A. K. Swain and A. S.Morris. (2004). Dynamic Control of Multi-arm Cooperating Manipulators Systems. Robotic. 2004, Vol.22, No.271-283.

K. Yoshida, R. Kurazume and Y. Umetani. (1991). Dual Arm Coordination in Space Free-Flying Robot. Proc. IEEE Conf. Rob. Automat, 1991, 2516-2521.

S. Ali A et al. (1997). Coordinated Motion Control of Multiple Manipulator Space Free-Flyers. $7^{\text {th }} A A S / A I A A$ Space Flight Mechanics Meeting, 1997, 10-12.

S. Ali A. Moosavian and E. Papadopoulos. (1997). On the Control of Space Free-Flyers Using Multiple Impedance Control. Proc. of the IEEE Conf. Rob. Automat, 1997, 853-858.

S. H. Murphy, J. T. Wen and G. N. Saridis. (1991). Simulation of Cooperating Robot Manipulators on a Mobile Platform. IEEE Trans. Rob. Automat, 1991, 7(4), 468-478.

Yan-Ru Hu and George Vukovich. Dynamic Control of Free-Floating Coordinated Space Robots. Journal of Robotic Systems, 1998, 15(4), 217-230.

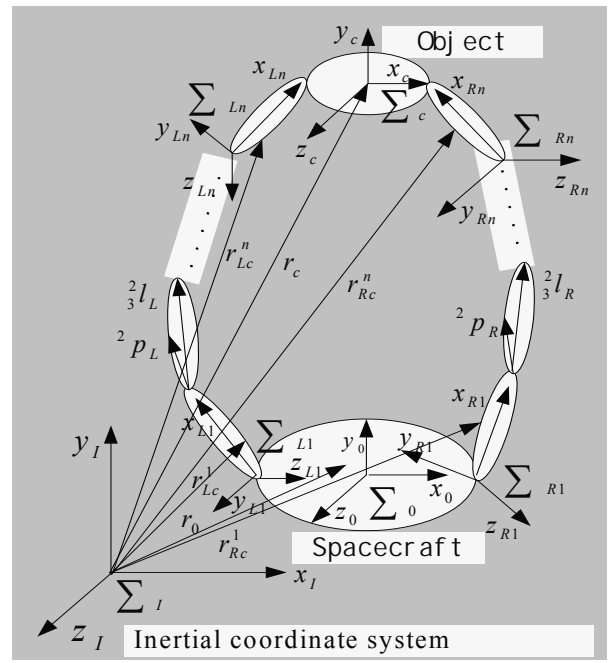

Figure 1. The grasp system for a free-floating space dual -arm robot 

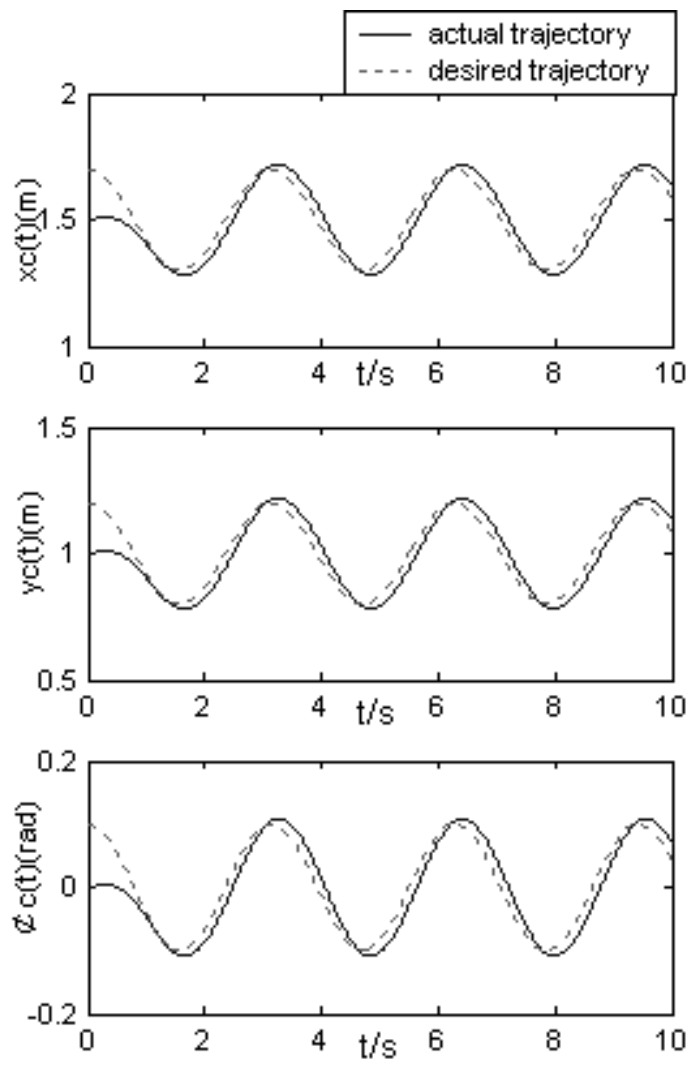

Figure 2. The tracking trajectories of the object
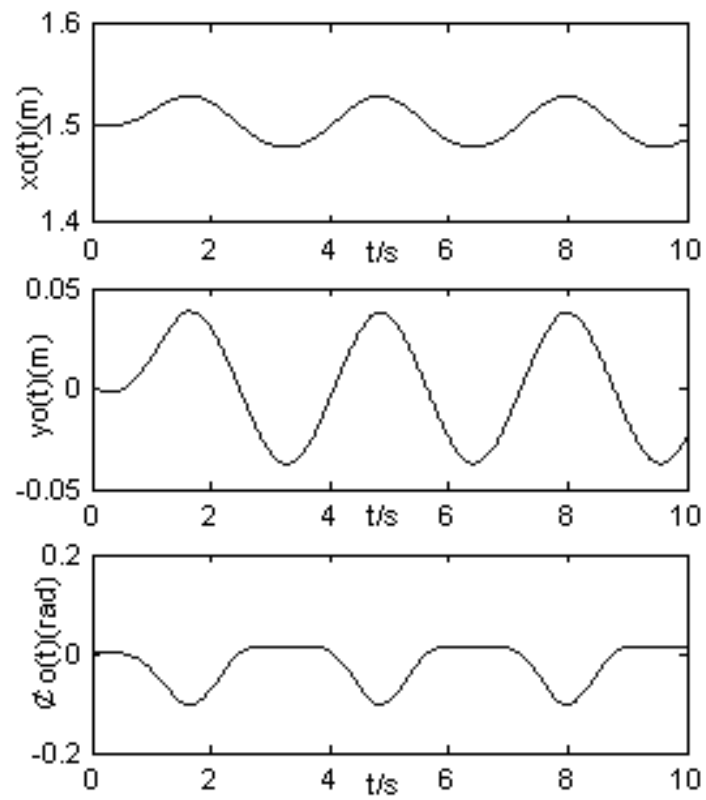

Figure 3. The movement of the spacecraft 


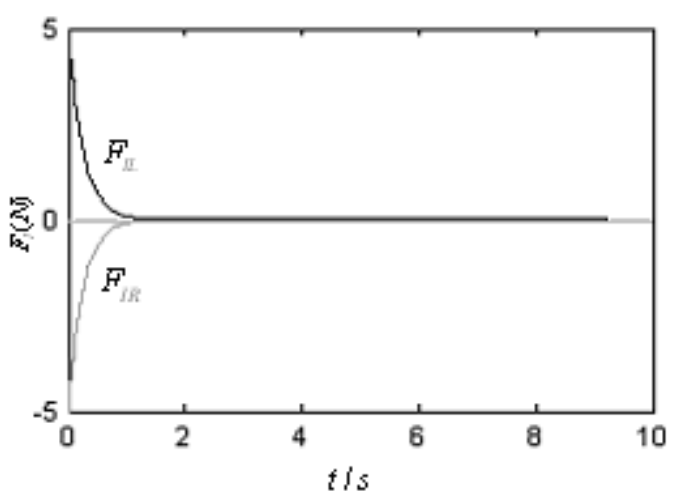

Figure 4. Zero internal force responses of the dual-arms

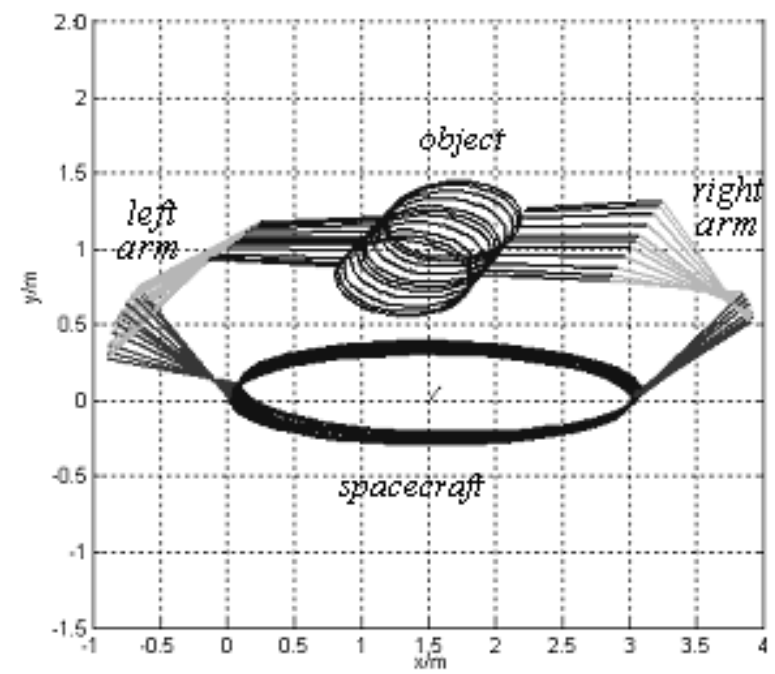

Figure 5. Motion responses of the dual-arms, object and spacecraft 